Research Round Up-COVID-19, Coagulopathy and Clotting Abnormalities

Introduction

The last research round up provided you with an overview of recent research end of life care in a global pandemic. In the last 9 months since the start of rapid publications around COVID-19 that I feared I may struggle to define a relevant topic for this months round up. However, as I was reading the information around coagulopathy in COVID-19 from a personal perspective it seemed appropriate to carry this further for the journal piece. There is emerging evidence that changes to coagulation and embolic event are linked with the more serious cases of COVID-19 and may be prognostic of outcome as these events contribute to morbidity and mortality. This month I am looking at 3 publications around the subject including one that explains the coagulation process to help contextualise the problem. There is little related to prescribing choices as initially low molecular weight heparin was used but now other anticoagulant medications are being trialled and hopefully results form these will be available in the near future.

\title{
Coagulopathy and COVID-19
}

This review article from Tokyo in Japan was published in March 2020 and outlined what was being seen by clinicians dealing with ill patients in the first wave of the COVID-19 pandemic. It was recognised fairly early on that many of the patients who were hospitalised with COVID-19 were also presenting with coagulopathy issues. This paper also outlines the coagulation process to highlight what the changes were doing to the normal clotting cascade mechanisms and how that made be used to understand what was compromised. The authors report that the most seriously ill patients with coronavirus exhibited clinical signs of varying thrombo-embolic conditions, including disseminated intravascular coagulation (DIC) like symptoms, pulmonary embolism and large intravascular embolism. They report that bleeding tendency and haemorrhage are rare but that end organ function is often affected. These changes can be seen clinically but also in altered blood and biochemical biomarkers. They are able to speculatively relate these biomarker changes to altered clotting cascade and end outcome events. There is a good discussion on coagulation therapy around both the need for it and choice of low molecular weight heparin as an initial intervention. This paper helps sets the scene as one of the earliest published discussion of hypercoagulopathy and COVID-19.

Iba, T., Levy, J.H., Levi, M \& Thachil, J. (2020) Coagulopathy and COVID-19, J Thromb Heamost 18:2103-2109 retrieved from https://onlinelibrary.wiley.com/doi/epdf/10.1111/jth.14975

\section{COVID-19 coagulopathy: An in-depth analysis of the coagulation system}

This original research paper was published in August 2020 reports on a study of 206 Spanish patients who presented with COVID-19. The aim of the study was to analyse the normal bloods taken and the coagulation parameters of patients with COVID-19, This was to determine whether coagulation factor derogation occurs and identify potential prognostic biomarkers of the disease. This was a retrospective study examining already collected data on patients hospitalised with COVID-19 between April 3rd and May 3rd 2020. Participant inclusion was determined by the following criteria; being a patient diagnosed with COVID-19, over18 years and, hospitalized. Anyone receiving Vitamin $\mathrm{K}$ therapy was excluded. COVID-19 diagnosis was defined by 'positive PCR in nasopharyngeal swab' 
or by 'radiologic and analytical findings highly suggestive of the disease'. The bloods that were taken were done according to the COVID-19 protocol in place in that hospital at the time of the study. These included red and white cell blood count, biochemistry, C-reactive protein, procalcitonin, ferritin, and interleukin 6 . In addition, D-dimer and coagulation factors were also analysed. Of the 206 participants, 18 died with 188 survivors. The main finding of the study was an elevated d-dimer with $69.4 \%$ of the patients' above normal range. Factor VII levels also showed an increasing trend but did not reach statistical significance. They conclude that abnormal coagulation and other routine laboratory parameters were associated with poor prognosis. Notably, D-dimer levels were significantly higher in non-survivors who also showed changes to prothrombin.

Martin-Rojas, R.M. etal (2020)_COVID-19 coagulopathy: An in-depth analysis of the coagulation system European Journal of Haematology, 105: 741-750 Retrieved from https://onlinelibrary.wiley.com/doi/epdf/10.1111/ejh.13501

\section{Endothelial Cells Orchestrate COVID-19 Coagulopathy}

This comment piece in the Lancet Haematology section links endothelial cells to covid-19 associated coagulopathy. They report that in COVID-19 patients that coagulation activation is widely seen and that post mortem reports have identified many cases of widespread microthrombi disseminated throughout the pulmonary vasculature, suggesting that vasculopathy is important in COVID-19 pathogenesis. These post-mortem studies also show substantial endothelial cell damage, with evidence of apoptosis (cell death) and loss of tight junctions. Collectively, these data suggest that endothelial cells play a key role in orchestrating the unusual pulmonary intravascular coagulopathy associated with severe acute respiratory syndrome coronavirus 2 (SARS-CoV-2) infection. There is a good discussion and visual interpretation of the potential activation cascade from endothelium to aid understanding. There is a good discussion around the significance of elevated Von Willebrand Factor (VWF) and disease severity with more patients who display this symptom being admitted to intensive care settings. VWF can bind to platelet receptors causing platelet adhesion and aggregation leading to hypercoagulability. Normal blood vessels are lined by an endothelial cell monolayer that plays a crucial role in preventing formation of pathological thrombosis. Thrombomodulin on endothelial cells has anticoagulant properties. If thrombomodulin is shed, which it can be due to cytokine activation, then this anticoagulant property of endothelial cells is compromised. They conclude that further studies will be required to define the different mechanisms through which SARS-CoV-2 infection causes such marked endothelial cell effects and how this can be ameliorated or managed pharmacologically.

O'Sullivan, J.M., McGonagle, D., Ward, S.E., Preston, R.J.S. \& O'Donnell, J.S. 2020 Endothelial Cells Orchestrate COVID-19 Coagulopathy. Lancet Haematology Volume 7, Issue 8, E553-555

https://www.thelancet.com/action/showPdf?pii=S2352-3026\%2820\%2930215-5 


\section{Conclusion}

There seems to be a growing body of evidence to support potentially significant numbers of patients who contract COVID-19 having some vascular and coagulopathy symptoms which may or may not cause morbidity and mortality. These range from simple elevated d-dimer with no clinical correlation to patients experiencing fatal embolic events. What is evident is that this is an area for significant further research and coagulopathy should be assessed for in all patients presenting with symptomatic COVID-19 and appropriate anticoagulation therapy prescribed to prevent significant embolic events. 